\title{
'Migrating' intraventricular neurocysticercus cyst
}

\author{
S J Przybojewski, MB ChB, Dip Pec (SA), \\ MMedRad (D), FCRad (D) \\ $S$ B Griffith-Richards, $M B C h B$ \\ M Strachan, MB ChB, LMCC \\ Y Vadachia, MB ChB, MMed Rad (D) \\ D L Kathan, MB ChB, MSc, MBA
}

Department of Radiology, Stellenbosch University and Tygerberg Hospital

\section{Abstract}

This report describes an intraventricular neurocysticercus cyst of the 4th ventricle with consequent hydrocephalus in an adult man. He was managed with anti-helminthic therapy and a 3rd ventriculostomy. Initial imaging showed the cyst within the 4th ventricle. At follow-up imaging a cyst was present in the 3 rd ventricle and the 4 th ventricle had returned to normal. It is postulated that the cyst 'migrated' from the 4th to the 3rd ventricle.

\section{Introduction}

Neurocysticercosis (NCC) is more commonly seen to involve the brain parenchyma and uncommonly involves the ventricular system and subarachnoid spaces. ${ }^{1}$ Whereas parenchymal cysticerci can be readily identified on CT and MRI, most authors agree that it is difficult to identify lesions within the cerebrospinal fluid (CSF) spaces. ${ }^{1}$ We present a case of NCC of the 4th ventricle which was well demonstrated on conventional MRI sequences. On follow-up imaging the cyst appeared to have 'migrated' to the 3rd ventricle. We are not aware of any reports in the English literature describing 'migrating' intraventricular NCC cysts.

\section{Case description}

A 24-year-old male patient presented with progressively worsening occipital headaches and diplopia over a 4-month period. Routine observations were normal and neurological examination was unremarkable. Specifically, there were no cerebellar signs, signs of meningism or papilloedema. A CT scan (Fig. 1) showed hydrocephalus with a disproportionately enlarged 4 th ventricle. In addition, multiple calcified punctate lesions were seen scattered throughout the brain parenchyma in keeping with old NCC lesions. There was no abnormal contrast enhancement. The patient was seen by the neurosurgeons who managed the patient as having hydrocephalus of uncertain origin. A 3rd ventriculostomy was performed and the patient responded well. MRI (Fig. 2) showed resolution of the hydrocephalus post $3 \mathrm{rd}$ ventriculostomy and a 4 th ventricular cyst of CSF signal intensity on T1 and T2-weighted sequences. The cyst wall was best demonstrated on the axial and sagittal FLAIR sequences. Furthermore, an eccentric FLAIR hyperintense mural nodule was easily identified. This nodule represented the scolex and the diagnosis of intraventricular NCC was made. The patient was discharged on albendazole and prednisone. The patient was asymptomatic at follow up 3 months later and repeat MRI (Fig. 3) showed that the 4th ventricle had returned to its normal size and no longer contained the previously seen cyst.

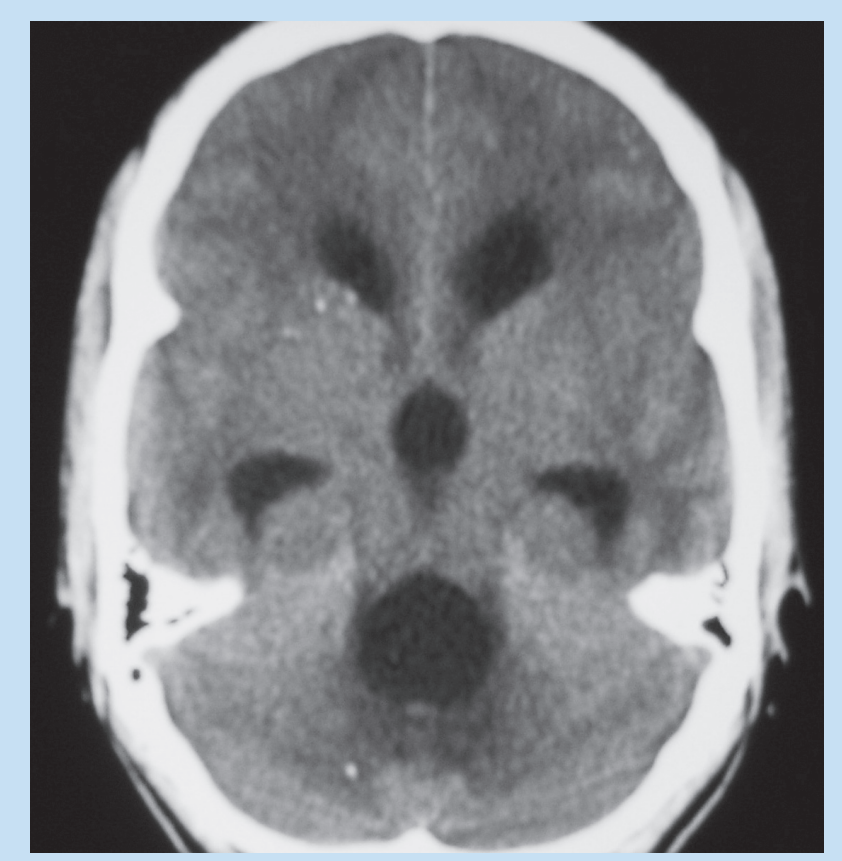

Fig. 1. Uncontrasted CT scan of the brain showing marked hydrocephalus, a disproportionately enlarged 4th ventricle, loss of surface sulcal markings and compression of the basal cisterns. Multiple punctate calcified parenchymal lesions are also present, consistent with old NCC.

However, a cyst with an eccentric scolex was now present within the 3 rd ventricle. It is postulated that the 4th ventricular cyst had 'migrated' to the 3 rd ventricle.

\section{Discussion}

NCC is an infestation by the larval form of Taenia solium and is the most frequently encountered parasite of the CNS. ${ }^{2}$ It is endemic in the developing world ${ }^{2}$ and contributes significantly to acquired epilepsy. ${ }^{1,3}$ The incidence is increasing in the developed world as a result of widespread international travel to endemic regions and immigration of infected individuals. ${ }^{1,3}$ Upon ingestion of infected meat, viable eggs adhere to and penetrate the gastrointestinal mucosa to enter the circulation..$^{1-3}$ NCC occurs when cysticercus larvae infect the central nervous system. ${ }^{1}$ The larvae normally land in the brain parenchyma and less commonly lodge in the ventricles and the CSF cisterns. ${ }^{1,4}$ Cysts can also be encountered in several locations at once. ${ }^{2,45}$

NCC can be asymptomatic or present with a variety of clinical manifestations including convulsions and signs and symptoms of raised intracranial pressure. ${ }^{1,2}$ Presentation depends on several factors such as host response, stage of evolution, and number, size and location of cysticerci. ${ }^{1,2,5}$ Patients with parenchymal cysts normally present with seizures, whereas patients with intraventricular cysts usually present with headaches, vomiting and visual disturbances as a result of hydrocephalus which is potentially fatal. ${ }^{1,2,5,6}$ The 4 th ventricle is the most common intraventricular site $e^{2,5}$ probably because of gravity or direct entry via the choroid plexus. ${ }^{6}$ Cysts can degenerate in the ventricular system causing an ependymal reaction identifiable on contrast-enhanced MRI and can lead to permanent neurological complications. ${ }^{2}$ 


\section{CASE REPORT}
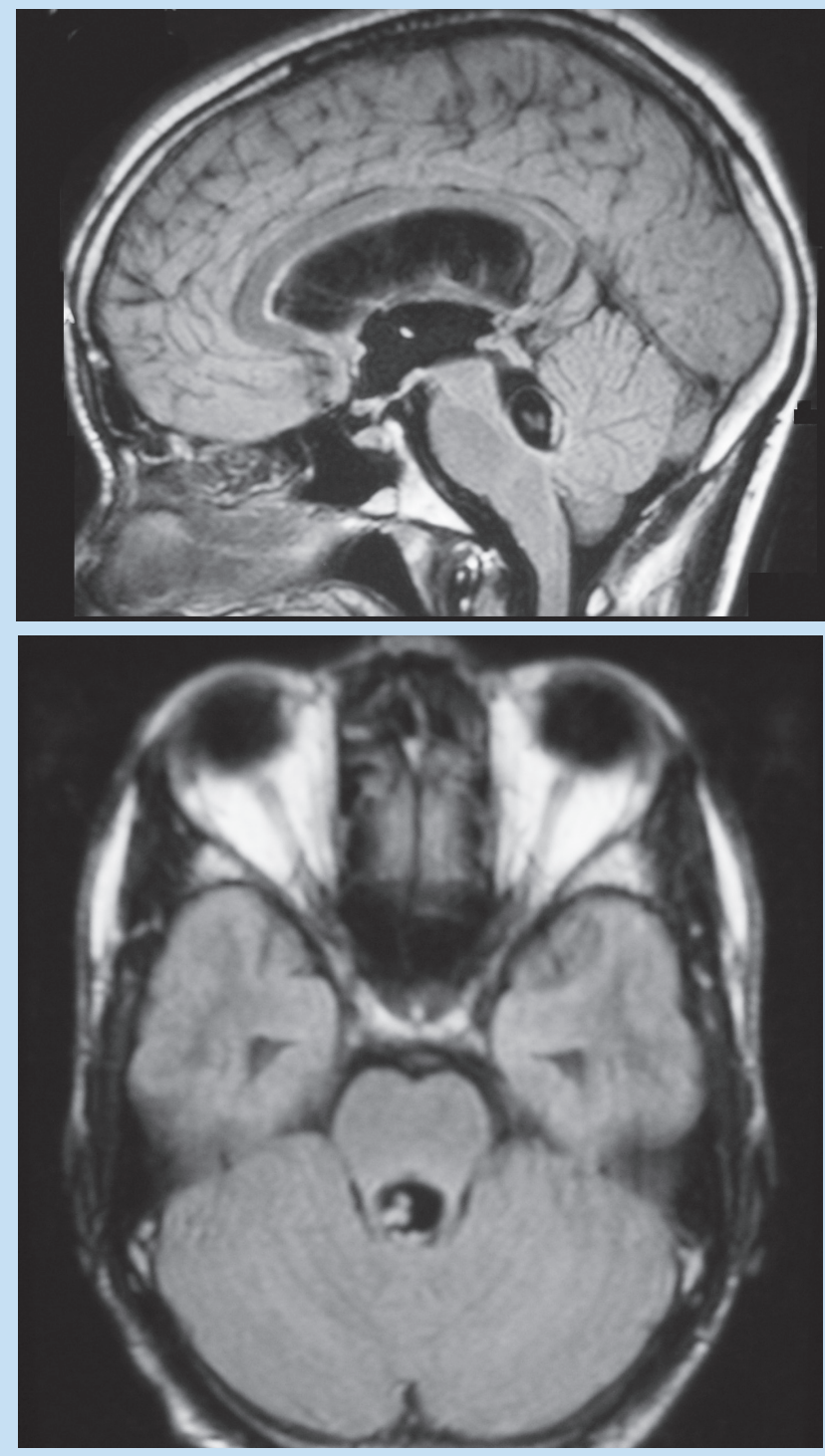

Figs. 2a and b. Sagittal and axial FLAIR MR images clearly demonstrating the 4th ventricular neurocysticercus cyst and the eccentric scolex. The hydrocephalus is markedly improved post-3rd ventriculostomy.

Before the advent of MRI, intraventricular cysts were difficult to diagnose noninvasively. ${ }^{2}$ Previously used invasive procedures included contrast and CT ventriculography. ${ }^{2}$ MRI is currently the most useful imaging tool for $\mathrm{NCC}^{4,5}$ and is superior to $\mathrm{CT}^{2,5}$ It is especially useful for the assessment of intraventricular cystic lesions and is often diagnostic. ${ }^{2}$ However, unlike parenchymal NCC, it is often not possible to detect NCC cysts when they are present in the CSF spaces ${ }^{1}$ using conventional MRI sequences as they are obscured by the adjacent CSF. $^{2,3}$ Despite this, neuroimaging remains one of the most important clinical tools for the diagnosis of NCC. ${ }^{4}$ T1-weighted sequences are reportedly more sensitive than T2-weighted sequences. ${ }^{2}$ Proton density images may also better delineate cysts from surrounding CSF. ${ }^{5}$ Other sequences reported to be useful include three-dimensional (3D) spoiled gradient recalled echo $(\mathrm{SPGR})^{1}$ and $3 \mathrm{D}$ constructive interference in steady state (3D-CISS)
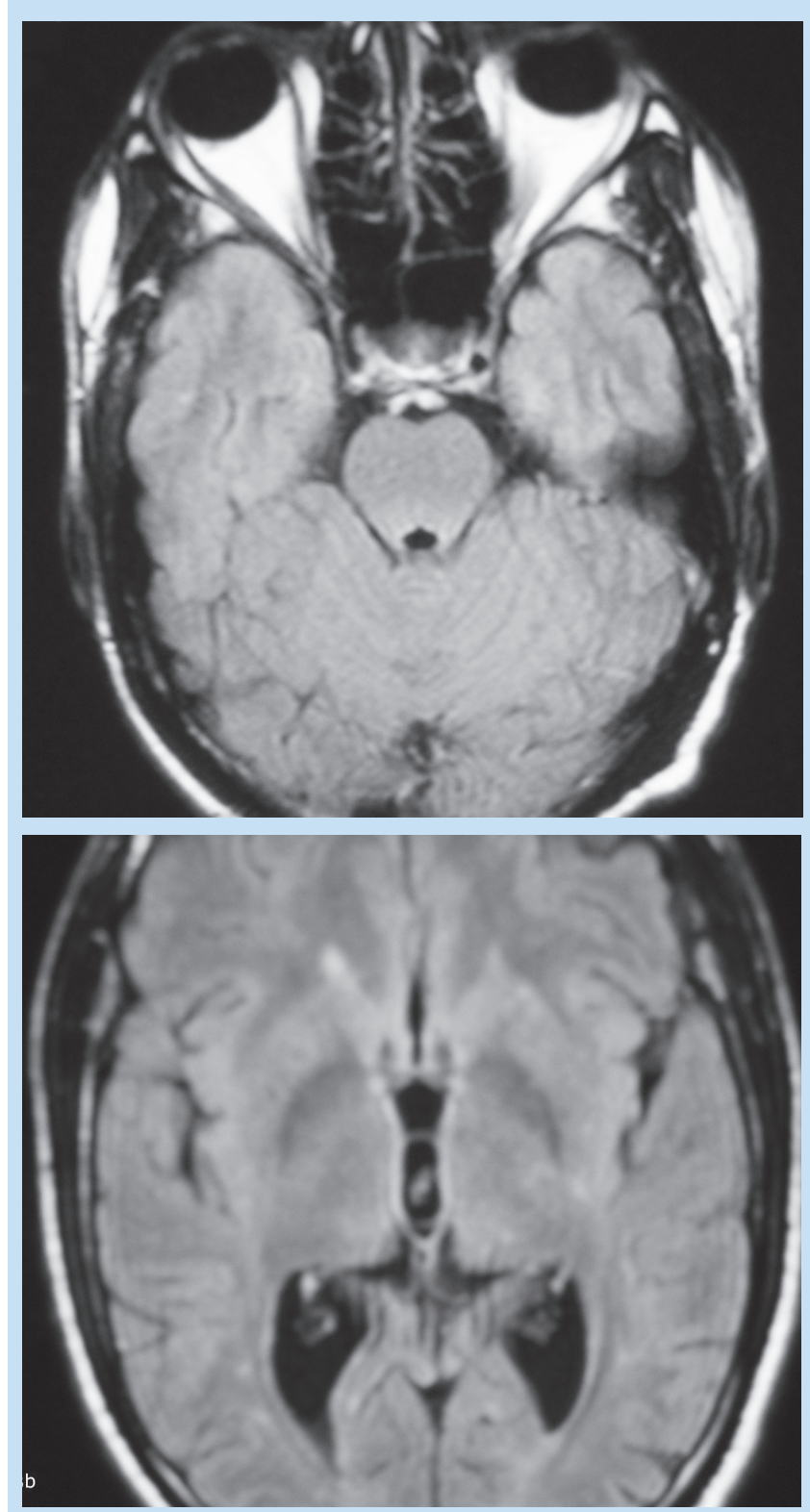

Figs. 3a and b. Follow-up axial FLAIR MR images showing a 3rd ventricular neurocysticercus cyst and scolex not present at initial MRI. The previously abnormal 4 th ventricle has returned to its normal configuration.

which is a heavily T2-weighted high-resolution sequence. ${ }^{2}$ Degenerating cysts can be detected on FLAIR and contrast-enhanced T1-weighted sequences by their increased signal intensity. ${ }^{3}$ FLAIR sequences proved to be the most useful in our patient.

Management of NCC depends on the area of brain involvement. ${ }^{3}$ Many authors recommend surgical excision as the treatment of choice for intraventricular NCC before the cysts degenerate causing ventriculitis ${ }^{1,2,4,6}$ and irreversible hydrocephalus. ${ }^{1,4}$ Other authors advocate a trial of anti-helminthic therapy ${ }^{4,7}$ and if hydrocephalus is present, shunt procedures or ventriculostomy should be performed, ${ }^{4}$ as was done in our patient. These patients should be monitored closely for recurrent hydrocephalus. ${ }^{2,4}$ 


\section{CASE REPORT}

Robbani I, Razdan S, Pandita KK. Diagnosis of intraventricular cysticercosis by magnetic resonance imaging: Improved detection with three-dimensional spoiled gradient recalled echo sequences. Australas Radiol 2004; 48:237-239.

2. Govindappa SS, Narayanan JP, Krishnamoorthy VM, et al. Improved detection of intraventricular cysticercal cysts with the use of three-dimensional constructive interference in steady state MR sequences. Am J Neuroradiol 2000; 21: 679-684.

3. Hauptman JS, Hinrichs C, Mele C, et al. Radiologic manifestations of intraventricular and subarachnoid racemose neurocysticercosis. Emerg Radiol 2005;11:153-157
Cuetter AC, Garcia-Bobadilla J, Guerra LG, et al. Neurocysticercosis: Focus on intraventricular disease. Clin Infect Dis 1997; 24:157-164

5. Ghosh D, Dubey TN, Prabhakar S. Brain parenchymal, subarachnoid racemose, and intraventricular cysticercosis in an Indian man. Postgrad Med J 1999;75:164-166.

6. Madrazo I, Garcia-Renteria JA, Sandoval M, et al. Intraventricular cysticercosis. Neurosurgery 1983; 12: 148-152.

Allcut DA, Coulthard A. Neurocysticercosis: regression of a fourth ventricular cyst with praziquantel. Neurol Neurosurg Psychiatry 1991; 54:461-462.

\section{Interventional Radiology Secrets}

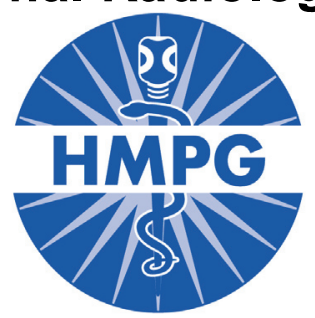

By David L. Waldman; Nikhil C. Patel and Wael E.A. Saad

ISBN 1560536098 / 9781560536093 - Paperback Hanley \& Belfus - Published September 2004

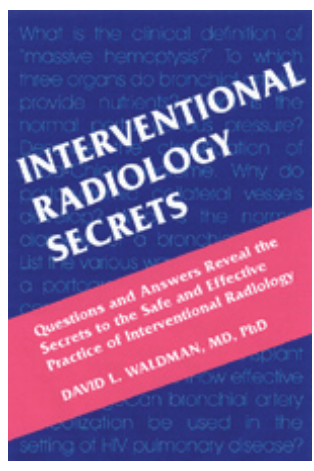

Health \& Medical Publishing Group

Private Bag X1, Pinelands, 7430

Tel: 021 - 6578200 - Fax: 0216834509

e-mail: carmena@hmpg.co.za / brents@hmpg.co.za

Noted expert Steven D. Waldman, MD, JD, is the editor of this volume in the Secrets Series on one of the hottest topics in the field-interventional radiology. He provides clear, concise coverage of all of the basics as well as succinct, but thorough coverage of both vascular and nonvascular topics. The Secrets popular question and answer format makes this title perfect for board review, as a clinical refresher, or as a quick-reference guide.

\section{Features}

- Hot topics include: gene therapy, noninvasive diagnosis and management of vascular disease, and cost-effective outpatient clinics.

- Offers guidance on all of interventional radiology s most important concepts in the proven Q\&A format of the Secrets Series $®$.

- Features the author s pearls, tips, memory aids, and secrets in the answer sections.

- Provides bulleted lists and algorithms for quick review.

- Presents a wealth of clear, crisp illustrations and images that bring the text to life. 